\title{
Interoperability Framework for Smart Home Systems.
}

\begin{abstract}
Recent advancements in smart home systems have increased the utilization of consumer devices and appliances in home environment. However, many of these devices and appliances exhibit certain degree of heterogeneity and do not adapt towards joint execution of operation. Hence, it is rather difficult to perform interoperation especially to realize desired services preferred by home users. In this paper, we propose a new intelligent interoperability framework for smart home systems execution as well as coordinating them in a federated manner. The framework core is based on Simple Object Access Protocol (SOAP) technology that provides platform independent interoperation among heterogeneous systems. We have implemented the interoperability framework with several home devices to demonstrate their effectiveness for interoperation. The performance of the framework was tested in Local Area Network (LAN) environment and proves to be reliable in smart home setting
\end{abstract}

Keyword: Interoperability; Smart home; SOAP; Web services 